\title{
RKKY Interaction in a Chirally Coupled Double Quantum Dot System
}

\author{
A. W. Heine*, D. Tutuc*, G. Zwicknagl ${ }^{\dagger}$, D. Schuh ${ }^{* *}$, W. Wegscheider ${ }^{\ddagger * * *}$ and \\ R. J. Haug* \\ *Institut für Festkörperphysik, Leibniz Universität Hannover, Appelstr. 2, 30167 Hannover, Germany \\ $\dagger$ Institut für Mathematische Physik, TU Braunschweig, Mendelssohnstr. 3, 38106 Braunschweig, Germany \\ ** Institut für Experimentelle und Angewandte Physik, Universität Regensburg, Universitätstr. 31, 93053 \\ Regensburg, Germany \\ ${ }^{\ddagger}$ Laboratorium für Festkörperphysik, ETH Zürich, Schafmattstr. 16, 8093 Zürich, Switzerland
}

\begin{abstract}
The competition between the Kondo effect and the Ruderman-Kittel-Kasuya-Yoshida (RKKY) interaction is investigated in a double quantum dots system, coupled via a central open conducting region. A perpendicular magnetic field induces the formation of Landau Levels which in turn give rise to the so-called Kondo chessboard pattern in the transport through the quantum dots. The two quantum dots become therefore chirally coupled via the edge channels formed in the open conducting area. In regions where both quantum dots exhibit Kondo transport the presence of the RKKY exchange interaction is probed by an analysis of the temperature dependence. The thus obtained Kondo temperature of one dot shows an abrupt increase at the onset of Kondo transport in the other, independent of the magnetic field polarity, i.e. edge state chirality in the central region.
\end{abstract}

Keywords: Quantum Dots, Kondo effect, RKKY interaction

PACS: 73.21.La, 73.23.Hk, 73.63.Kv

Lateral Quantum Dots (QDs) have attracted a great deal of attention from the scientific community due to their high versatility and tunability. Since the spin of a confined electron in a QD was proposed as an implementation of a quantum bit (qubit) in a solid states system [1], the investigation of single, double or triple QDs has led to many breakthroughs, for instance the observation of the Kondo effect $[2,3,4]$ - a signature of spin entanglement in a many-body system where delocalized electrons screen a localized spin. Furthermore, long range electron mediated spin-spin exchange interactions, like the Ruderman-Kittel-Kasuya-Yoshida (RKKY) interaction, could provide a solution to spin entanglement beyond the nearest-neighbor restraint.

At very low temperatures, in a QD with a non-zero total spin, i.e. odd number of electrons, the Kondo effect leads to the formation of a singlet ground state between the spin on the dot and a conduction electron in the leads. The new ground state induces the formation of a peak in the density of states which basically allows transport through the QD in a situation where sequential tunneling events are otherwise Coulomb-blocked. The energy scale of the interaction is described by the so-called Kondo temperature $T_{K}$ [2]. The Kondo impurities interact also with one-another via carrier mediated spin-spin interactions, like the RKKY, which compete with the local interaction that give rise to the Kondo effect [5, 6, 7, 8, 9]. In the present work we use transport measurements of the

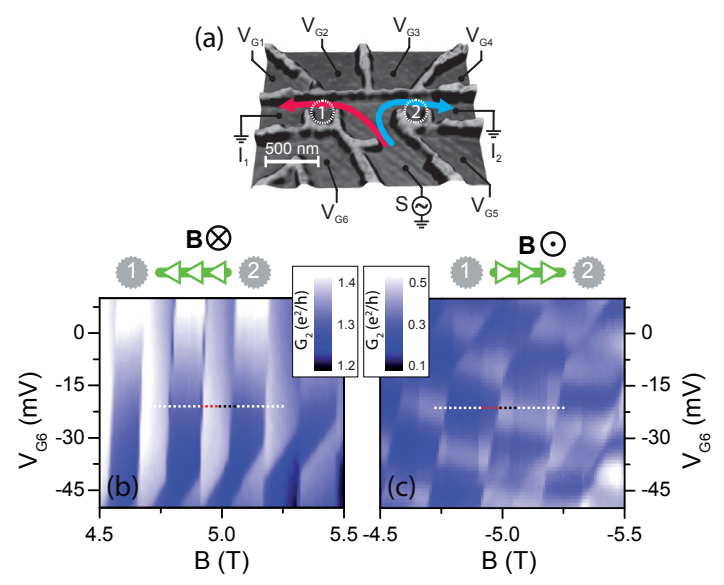

FIGURE 1. (a) AFM image of our device defined by oxide lines (bright). Quantum dots, 1 and 2, are connected to a common source $S$, and each to individual drains. Six in-plane gates, G1-G6, control the potentials of the dots and coupling to the leads. Differential conductance $G_{2}$ through QD2 as a function of $V_{G 6}$ and magnetic field, for positive (b) and negative (c) magnetic field polarity. Differential conductance $G_{2}$ through QD2 as a function of $V_{G 6}$.

Kondo effect as a spectroscopic tool to probe the presence of the RKKY interaction.

The sample consists of two lateral QDs (Fig. 1 (a)) 

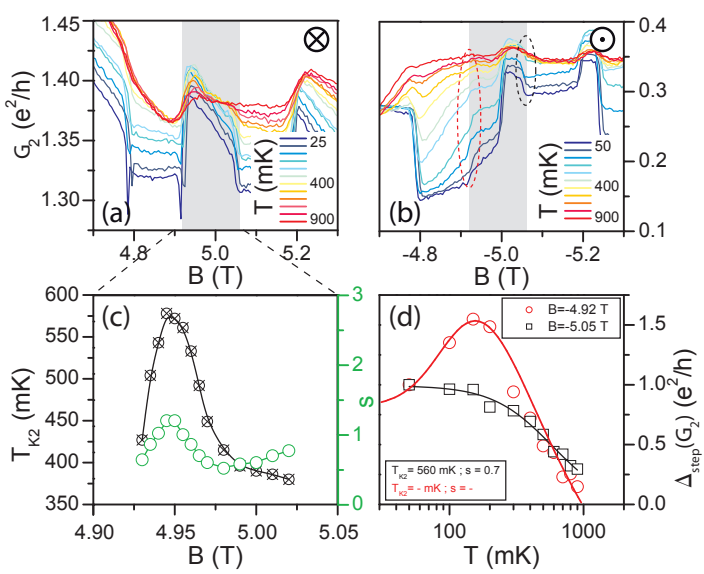

FIGURE 2. Temperature dependence of QD2 for (a) positive and (b) negative magnetic field polarity, taken along the dashed lines in Fig. 1(b), and 1(c) respectively. (c) $T_{K 2}(\otimes)$ vs magnetic field and the $s$ fitting parameter (o). (d) Temperature dependence with respect to the background at the corresponding positions in (b).

defined by local anodic oxidation with an AFM on a GaAs/AlGaAs heterostructure containing a 2DEG $36 \mathrm{~nm}$ bellow the surface. The QDs are connected to a common Source via an open conducting region, and individual Drains. Further details about the sample and measurement setup can be found in [9]. A magnetic field applied perpendicular to the 2DEG induces the formation of Landau levels in the QDs which give rise to the so-called Kondo chessboard [10, 11, 12, 13]. The edge states formed in the central open conducting region will couple the QDs chirally [9], and the coupling direction can be controlled by the magnetic field polarity. In the measurements of e.g. QD2 this effect is seen as a clean chessboard when the QD is "up-stream", i.e. positive magnetic field (Fig. 1 (b)), or has superimposed the negative of the QD1 chessboard when it's "downstream", i.e. negative magnetic field (Fig. 1 (c)).

The presence of the RKKY exchange interaction in QD2 is probed by analyzing the temperature dependence of the Kondo transport in the $-4.92 \mathrm{~T}$ to $-5.05 \mathrm{~T}$ interval, as well as $4.92 \mathrm{~T}$ to $5.05 \mathrm{~T}$ (gray regions in Fig. 2 (a) and (b)). From the Kondo chessboard measurements, we also know that QD1 shows Kondo transport between -4.77 to $-4.97 \mathrm{~T}$, respectively 4.77 to $4.97 \mathrm{~T}$ [9], therefore in the interval $-4.92 \mathrm{~T}$ to $-4.97 \mathrm{~T}$, respectively $4.92 \mathrm{~T}$ to $4.97 \mathrm{~T}$, both QDs are acting as Kondo impurities. $T_{K 2}$, i.e. the Kondo temperature of QD2, is extracted from the measurements shown in Fig. 2 (a) using $G(T)=$ $G_{0}\left(T_{K 2}^{\prime} /\left(T^{2}+T_{K 2}^{\prime}\right)\right)^{s}$ with $T_{K 2}^{\prime}=T_{K 2} /\left(\sqrt{2^{1 / s}-1}\right)$ [3], after subtracting a polynomial background for each temperature trace. The obtained result is shown in Fig. 2 (c), where $T_{K 2}$ exhibits an abrupt increase and decrease in the magnetic field range where the Kondo transport regions in both QDs overlap, a result very similar to the one obtained for QD1 [9].

For the negative magnetic field situation, when QD2 is "downstream" from QD1, the non-monotonic background makes a similar extraction of the Kondo temperature over the whole interval unreliable. However an analysis of the conductance steps with respect to the background at the onset of Kondo transport, i.e. at $-4.92 \mathrm{~T}$ and $-5.05 \mathrm{~T}$, is possible. By using an averaged difference filter on each temperature trace in Fig. 2 (b) we obtain the conductance step height $\Delta_{\text {step }}\left(G_{2}\right)$, shown in Fig. 2 (d). While the temperature dependence of the step height at $-5.05 \mathrm{~T}$ shows the expected logarithmic drop with temperature and can be fitted with Eq. (2) in Ref. [3], the step height at $-4.92 \mathrm{~T}$ shows a highly non-monotonic behavior with temperature. The obvious qualitative difference between the two traces, indicates again that the two Kondo transport regions are characterized by different ground states.

Both the abrupt increase and decrease in $T_{K 2}$ shown in Fig. 2 (c) and the non-monotonic conductance stepheight behavior with temperature in Fig. 2 (d) are correlated with the onset of Kondo transport in QD1 and are interpreted in terms of RKKY exchange interaction between the spins of the QDs via the edge states in the central region. Although in the case of QD2 the effect of the edge state transport direction is stronger, it is still unclear what role the chirality of the edge states is playing in the exchange interaction between the two spins.

This work has been supported by German Excellence Initiative via QUEST and the NTH School for Contacts in Nanosystems.

\section{REFERENCES}

1. D. Loss, D. P. DinVincenzo, Phys. Rev. A 57, 120 (1998).

2. D. Goldhaber-Gordon et al., Nature 391, 156 (1998).

3. D. Goldhaber-Gordon et al., Phys. Rev. Lett. 81, 5225 (1998).

4. S. M. Cronenwett, T. H. Oosterkamp, L. P. Kouwenhoven, Science 281, 540 (1998)

5. N. J. Craig et al., Science 204, 565 (2004).

6. P. Simon, R. López, Y. Oreg, Phys. Rev. Lett. 94, 086602 (2005).

7. M. G. Vavilov, L. I. Glazman, Phys. Rev. Lett. 94, 086805 (2005).

8. S. Sasaki et al., Phys. Rev. B 73, 161303 (2006).

9. D. Tutuc et al., Phys. Rev. B 83, 241308 (2011).

10. C. Fuehner et al., Phys. Rev. B 66, 161305 (2002).

11. M. Stopa et al., Phys. Rev. Lett. 91, 046601 (2003).

12. D. Kupidura et al., Phys. Rev. Lett. 96, 046802 (2006).

13. M. C. Rogge, C. Fuehner, R. J. Haug, Phys. Rev. Lett. 97, 176801 (2006) 
AIP Conference Proceedings is copyrighted by AIP Publishing LLC (AIP). Reuse of AIP content is subject to the terms at: http://scitation.aip.org/termsconditions. For more information, see http://publishing.aip.org/authors/rights-and-permissions. 